Alloys and Compounds

Elsevier Editorial System(tm) for Journal of

Manuscript Draft

Manuscript Number: JALCOM-D-19-03619

Title: In-situ Mo nanoparticles strengthened CoCrNi medium entropy alloy

Article Type: Full Length Article

Keywords: CoCrNi MEAs; Composites; Coating method; Spark plasma

sintering; Microstructure; Mechanical properties

Corresponding Author: Dr. Hailin Yang, Ph.D.

Corresponding Author's Institution: Powder Metallurgy Research Institute

First Author: Jianying Wang

Order of Authors: Jianying Wang; Hailin Yang, Ph.D.; Hua Huang; Jianying

Ruan; Shouxun Ji

Abstract: In present work, CoCrNi/Mo mixed powder was designed and fabricated by a novel coating method followed by the calcination and reduction processes. The results indicated that in-situ Mo particles distributed homogeneous among the CoCrNi gas atomized powder and no other drawbacks generated such as contamination and oxidation, the reduced Mo particles were nanoscale, and the maximum size did not exceed $\sim 600 \mathrm{~nm}$. The SPSed CoCrNi/Mo composite with significant amount of in-situ $\mu$ phases showed improved mechanical properties: the yield strength and hardness from $352 \mathrm{MPa}$ and $159 \mathrm{HV}$ to $815 \mathrm{MPa}$ and $375 \mathrm{HV}$, compared with the pure CoCrNi MEA. The improved properties are mainly attributed to synergistic effects of various strengthening mechanisms, including solid solution strengthening, load transfer effect, orowan strengthening, grain refinement, especially thermal mismatch mechanism. In addition, this could give insights on the applications of other MEA or HEA-based composites fabricated by coating method. 


\section{Prime novelty statement}

CoCrNi/Mo mixed powder was designed and fabricated firstly by a novel coating method followed by the calcination and reduction processes. In-situ Mo nanoparticles distributed homogeneous among the CoCrNi gas atomized powder and no other drawbacks generated such as contamination and oxidation. The SPSed CoCrNi/Mo composite with significant amount of in-situ $\mu$ phases showed superior mechanical properties, compared with the pure CoCrNi MEA. The improved properties are mainly attributed to synergistic effects of various strengthening mechanisms, especially thermal mismatch mechanism. In addition, this could give insights on the applications of other MEA or HEA-based composites fabricated by coating method. 


\title{
In-situ Mo nanoparticles strengthened CoCrNi medium entropy alloy
}

Jianying Wang ${ }^{1}$, Hailin Yang ${ }^{1 *}$, Hua Huang ${ }^{1}$, Jianming Ruan ${ }^{1}$, Shouxun $\mathrm{Ji}^{2}$

1 - State Key Laboratory of Powder Metallurgy, Central South University, Changsha 410083, China

2 - Institute of Materials and Manufacturing, Brunel University London, Uxbridge, Middlesex UB8 3PH, United Kingdom

* Corresponding author: Hailin Yang, E-mail address: y-hailin@csu.edu.cn

\begin{abstract}
In present work, CoCrNi/Mo mixed powder was designed and fabricated by a novel coating method followed by the calcination and reduction processes. The results indicated that in-situ Mo particles distributed homogeneous among the CoCrNi gas atomized powder and no other drawbacks generated such as contamination and oxidation, the reduced Mo particles were nanoscale, and the maximum size did not exceed $\sim 600 \mathrm{~nm}$. The SPSed CoCrNi/Mo composite with significant amount of in-situ $\mu$ phases showed improved mechanical properties: the yield strength and hardness from $352 \mathrm{MPa}$ and $159 \mathrm{HV}$ to $815 \mathrm{MPa}$ and $375 \mathrm{HV}$, compared with the pure CoCrNi MEA. The improved properties are mainly attributed to synergistic effects of various strengthening mechanisms, including solid solution strengthening, load transfer effect, orowan strengthening, grain refinement, especially thermal mismatch mechanism. In addition, this could give insights on the applications of other MEA or HEA-based composites fabricated by coating method.
\end{abstract}

Keywords: CoCrNi MEAs; Composites; Coating method; Spark plasma sintering; Microstructure; Mechanical properties 


\section{Introduction}

Medium entropy alloys (MEAs), a similar design strategy of high entropy alloys (HEAs, more than 5 kinds of elements) and consisting of two to four elements in equiatomic or near-equiatomic elements, have drawn much attention due to their attractive mechanical response [1-5]. In contrast to HEAs, MEAs are reported to have superior strength-toughness properties. For instance, the quaternary $\mathrm{Fe}_{50} \mathrm{Mn}_{30} \mathrm{Cr}_{10} \mathrm{Co}_{10}$ MEAs delivered higher yield strength and elongation in counterpart with the FeMnNiCoCr HEAs [6], and the quaternary TiZrNbTa MEAs showed 4 times better ductility than the quinary TiZrNbTaMo HEAs [5]. Moreover, superior comprehensive mechanical properties were achieved in the three-component CoCrNi MEAs [7-10], which two of the elements have been removed from FeMnCrNiCo HEAs, owing to steady strain hardening provided by dislocation activity and deformation-induced nano-twinning. This system of MEA, in turn, exhibited comprehensive strength-ductility properties that exceed those of most high entropy alloys and multi-phase alloys [7]. However, excellent mechanical properties of these MEAs with only single phase, in particular for face center cubic (FCC) crystal structure, may not sufficient in the industrial application where strength and hardness in the key requirement $[8,11]$. Thus, the increasing demand for better MEAs' properties has given rise to requirement of new approaches.

An alternative method of strengthening HEAs and MEAs involves reasonable component designs. For example, a novel concept of eutectic HEA (FCC/B2 phases) can be designed by alloy composition of $\mathrm{AlCoCrFeNi}_{2.1}$ with an unprecedented combination of castability, high fracture strength and high ductility [12]. Similarly, through generating dual-phase (FCC and hexagonal closed-packed (HCP)), outstanding strength-ductility trade-off was obtained in the $\mathrm{Fe}_{80-\mathrm{x}} \mathrm{Mn}_{\mathrm{x}} \mathrm{Cr}_{10} \mathrm{Co}_{10}$ (at.\%) non-equiatomic MEAs [6, 13-14]. Apart from component design, addition of enhanced particles to form coherent second phase or precipitate dispersion is another effective approach for adjusting comprehensive mechanical properties, such as Al [15], $\mathrm{Nb}[16], \mathrm{Mo}[17,18], \mathrm{Ti} / \mathrm{Al}[19,20]$ additions or other $\mathrm{SiC}$ [21], TiC [22] and $\mathrm{B}_{4} \mathrm{C}$ [23] 
ceramic particles. However, currently, introducing enhanced particles into MEAs and HEAs relies on conventional mechanical alloying or ball milling. The mixed powder prepared by these techniques can easily be contaminated and oxided by milling materials due to long time of high energy milling. Raza et al. [24] introduced Al to the CrFeMoV MEA by mechanical alloying (MA) and reported that a large number of $\mathrm{Al}_{2} \mathrm{O}_{3}$ particles were formed during the MA of the powder due to the very fine particle size and high surface energy. Similarly, oxides have been formed in the microstructure of the $\mathrm{CoCrNi} / \mathrm{B}_{4} \mathrm{C}$ composite as a consequence of powder contamination by oxygen [23].

To our best knowledge, coating method, which would eliminate unnecessary mechanical energy and porosity of powder, can be applied to synthesize mixed powder, compared to the long-time ball milling method. For instance, the WC-Co cemented carbides powder can be successfully prepared though chemical coating method [25, 26], near-spherical cobalt particles with a nano-size (200-400 nm) homogeneously distributed among the WC particles $(\sim 4.08 \mu \mathrm{m})$ after hydrogen reduction, in addition, XRD results indicated that the higher crystallinity of the WC-Co mixed powder fabricated through this route. Similarly, Chen et al. [27] synthesized the magnetic $\mathrm{Fe}_{3} \mathrm{O}_{4} @ \mathrm{TiO}_{2}$ nanocomposite by in-situ coating process at low temperature, using titanyl organic compounds as the precursor. Clearly, coating method has unique advantages in synthesizing the mixed powder in counterpart with traditional methods.

Therefore, in this present study, Mo-coated CoCrNi mixed powder was designed and synthesized by coating method followed by the calcination and reduction processes. Molybdenum salt particles, which were chosen as precursors, capped gas atomization CoCrNi powder and $\mathrm{CoCrNi} / \mathrm{Mo}$ mixed powder was obtained followed by calcining and reducing. In addition, corresponding CoCrNi MEAs and CoCrNi/Mo composites were fabricated by SPS. The microstructures and compositions of mixed powder and as-SPSed alloy were carefully characterized. Further, the strengthening mechanisms were systematically investigated and discussed. 


\section{Experimental methods}

\subsection{Starting matrials}

Commercial (NH4) ${ }_{2} \mathrm{Mo}_{4} \mathrm{O}_{13.2} \mathrm{H}_{2} \mathrm{O}$ (XiLong, Guangdong, China, purity $99.0 \%$ (mass volume), as a precursor in this study) and $\mathrm{CoCrNi}$ gas atomized powder were used as the starting materials in the experiments. $\mathrm{CoCrNi}$ powder in this work was prepared by the high-pressure nitrogen gas atomization with an operating temperature of 1943 $\mathrm{K}$ and a nozzle with a $\mathrm{N}_{2}$ pressure of $1.8 \mathrm{MPa}$.

\subsection{Powder and alloy manufacturing}

$\mathrm{CoCrNi} / \mathrm{Mo}$ mixed powder (mass fraction of Mo, $5 \%$ ) was prepared according to the method show schematically in Fig. 1. Firstly, $(\mathrm{NH} 4)_{2} \mathrm{Mo}_{4} \mathrm{O}_{13.2} \mathrm{H}_{2} \mathrm{O}$ precursor salts were dissolved into the deionized water at $60{ }^{\circ} \mathrm{C}, \mathrm{CoCrNi}$ gas atomized powder was then added into the solution to form CoCrNi-particle suspension under constant stirring at $120{ }^{\circ} \mathrm{C}$. Secondly, mixed powder was placed in vacuum at $60^{\circ} \mathrm{C}$ for $24 \mathrm{~h}$ after the deionized water has evaporated in suspension and mixed powder cannot be stirred further. Calcination was then operated at $500{ }^{\circ} \mathrm{C}$ under argon atmosphere followed by reduction in a tube furnace at $800{ }^{\circ} \mathrm{C}$ under hydrogen atmosphere. CoCrNi/Mo mixed powder was therefore obtained. Finally, CoCrNi gas atomized powder and $\mathrm{CoCrNi} / \mathrm{Mo}$ mixed powder were consolidated by SPS in a graphite die of an inner diameter of $40 \mathrm{~mm}$ at $1473 \mathrm{~K}$ for $15 \mathrm{~min}$ with a heating rate of $100 \mathrm{~K} / \mathrm{min}$ and a uni-axial pressure of $40 \mathrm{MPa}$.

\subsection{Microstructure characterization}

Phase constituents of powder and as-sintered samples were tested using X-ray diffraction (XRD, Rigaku X-2000) at $40 \mathrm{kV}$ with $\mathrm{Cu} K_{\alpha}$ radiation $\left(\lambda_{\mathrm{ka}}=1.54 \AA\right.$ A). Microstructures and phase compositions were examined using a scanning electron microscope (SEM, FEI nano 230 field emission) equipped with an energy-dispersive spectrometer (EDS). The grain size and preferred lattice was conducted at microscale using electron backscattered diffraction (EBSD) in a different SEM (hitachi S-3400 N). EBSD was equipped with an Oxford instrument Aztec HKL ${ }^{\circledR}$ imaging system. To distinguish the in-situ formed nanoprecipitates, further microstructure 
characterizations were performed using transmission electron microscope (TEM: Tecnai G2 F20). TEM samples were prepared by a combination method of the mechanical polishing and the precision ion polishing system (PIPS) at a voltage/an incident angle of $5 \mathrm{kV} / 3 \sim 8^{\circ}$.

\subsection{Evaluation of mechanical properties}

Dog-bone-shaped specimens with a gauge length of $10 \mathrm{~mm}$ and cross-section of $2.2 \times$ $1.5 \mathrm{~mm}^{2}$ were fabricated by electrical discharge machining for a tensile test. Both sides of the specimens were carefully ground to a 2000 grit finish using a $\mathrm{SiC}$ paper. The uniaxial tensile tests were performed using a material testing system (MTS Alliance RT30) tension machine at room temperature with an engineering strain rate of $1 \times 10^{-3} \mathrm{~s}^{-1}$. The hardness values of the CoCrNi MEA and CoCrNi/Mo composite samples were measured by an HVS-5 hardness tester. Also, a nanoindentation test was performed to measure the nanohardness employing UNHT equipment. All of the reported data are average values of at least 5 specimens.

\section{Results}

\subsection{Microstructure and chemical composition analysis of CoCrNi/Mo mixed powder}

XRD spectra of mixed powder at different states are indicated in Fig. 2a. The gas atomized powder only exhibits a single-phase FCC structure. After calnicing and reducing, the peaks of $\mathrm{MoO}_{3}$ and Mo were identified in the spectra of mixed powder, respectively. It is surmised that $\mathrm{MoO}_{3}(\mathrm{a}=3.9628 \AA, \mathrm{b}=13.855 \AA, \mathrm{c}=3.6964 \AA, \mathrm{Z}=$ $\left.2\left(\alpha=\beta=\gamma=90^{\circ}\right)\right)$ produced by calcining Mo salt, further transformed into Mo particles $\left(\mathrm{a}=3.1472 \AA, \mathrm{b}=3.1472 \AA, \mathrm{c}=3.1472 \AA, \mathrm{Z}=2\left(\alpha=\beta=\gamma=90^{\circ}\right)\right)$ after reducing and no other impurities generated. The detailed characterization of powder at different states, including morphology, dispersion and size, were further analyzed by SEM.

Fig. 3 presents SEM micrographs of CoCrNi gas atomized powder and mixed powder. The majority of the raw gas atomized powder displayed a spherical shape. Satellite particles were observed on the surfaces of a few of the powder due to the collision of the large and small powder particles during the flight process in the spray chamber 
(Figs. 3a and d). Mixed powder after calcining and reducing is shown in Figs. 3b-c and e-f. For mixed powder after calcining, $\mathrm{MoO}_{3}$ particles homogeneously distributed among the matrix powder, and almost all the surface of matrix powder were encased by $\mathrm{MoO}_{3}$ particles. Similarly, Mo particles homogeneously coated on the surface of matrix powder after reducing. The detailed individual morphology of mixed powder is shown in the Fig. 3f, the reduced Mo particles were nanoscale, and the maximum size did not exceed $\sim 600 \mathrm{~nm}$. Compared with traditional powder mixing methods, such as dry milling, wet milling in ethanol reported in different Refs [23, 28-29], the matrix powder particles in the mixed powder did not change obviously in their morphologies and maintain a spherical shape. Besides, the uniformity of Mo particle distribution was better than that of the effect of mechanical ball milling. EDS was further used for elemental mapping (Fig. 4) on the selected area of $\mathrm{CoCrNi} / \mathrm{Mo}$ mixed powder by coating method, the chemical compositions are summarized in Table 1. Co, $\mathrm{Cr}$ and $\mathrm{Ni}$ elements were distributed uniformly across the microstructure, the nanoparticles were composed of Mo elements and the distribution of oxygen elements rarely proved that the reduction effect of mixed powder was excellent.

\subsection{Microstructure of the sintered CoCrNi MEAs and CoCrNi/Mo composites}

XRD spectra of CoCrNi MEAs with 0 and 5 wt.\% Mo are shown in Fig. 2b. It is clear that the pure CoCrNi MEA has the single-phase FCC structure with no considerable change in the phase constituents, in contrast with CoCrNi powder. However, extra peaks of $\mu$ phase $\left(\mathrm{a}=2.85 \AA, \mathrm{b}=2.85 \AA, \mathrm{c}=4.554 \AA, \mathrm{Z}=85\left(\alpha=\beta=90^{\circ}, \gamma=120^{\circ}\right)\right)$ were also identified in the spectra of $\mathrm{CoCrNi} / \mathrm{Mo}$ composites. It is surmised that $\mu$ phases were originated from in-situ formation.

Fig. 5 shows the corresponding SEM morphologies of CoCrNi MEAs without and with 5 wt.\% Mo nanoparticles. As can be seen, as-SPSed CoCrNi MEAs appear to be a homogenous single phase with only few pores on the surface in Fig. 5a. In addition, annealing twins were observed in the microstructure of CoCrNi MEAs after electrolytic polishing (Fig. 5b). However, CoCrNi/Mo composites (Figs. 5c and d) show a significant amount of fine precipitates distributed among the microstructure. 
According to XRD data, these precipitates are in-situ $\mu$ phases. From higher magnification, nanoscale $\mu$ phases were mainly spherical and club shapes. To identify phase composition, chemical compositions of the FCC matrix and $\mu$ phase were calculated based on EDX measurement. It can be seen that the concentration of Mo and $\mathrm{Cr}$ were substantially higher in $\mu$ phase than in matrix from Table 1 .

The mean grain size and grain orientation of the CoCrNi MEAs and CoCrNi/Mo composites were studied using EBSD to understand the effect of in-situ $\mu$ phase on grain refinement of CoCrNi/Mo composites. As indicated by the EBSD maps in Fig. 6, with addition of Mo nanoparticles, an obvious grain refinement was observed in the composites. The grain size of FCC phase decreased from $20.80 \mu \mathrm{m}$ in CoCrNi MEAs to $8.64 \mu \mathrm{m}$ in $\mathrm{CoCrNi} / \mathrm{Mo}$ composites. The inverse pole figures (IPFs) of the FCC phase shown as inserts in Fig. 6 confirmed that the FCC grains have no obvious preferred orientation in CoCrNi MEAs and $\mathrm{CoCrNi} / \mathrm{Mo}$ composites.

In order to identify the microstructure and composition of CoCrNi MEAs and CoCrNi/Mo composites further. TEM bright field (BF) micrographs, selected-area diffraction patterns (SADPs) and EDS maps are shown in Figs. 7 and 8. Figs. 7a-c show that two phases were found in the microstructure of CoCrNi MEAs. SADPs analysis confirmed that there two type of phases were FCC matrix and twin phase. The formation of annealing twins revealed that the MEA system has a low stacking fault energy $\left(\gamma_{\mathrm{SFE}}\right)$ in the range of 18 to $45 \mathrm{MJ} \cdot \mathrm{m}^{-2}$ [21]. Figs. $7 \mathrm{~d}-\mathrm{f}$ show the EDS maps corresponding to area L1 in Fig. 8a. It is clear that the $\mathrm{Co}, \mathrm{Cr}$, and $\mathrm{Ni}$ elements were uniformly distributed, without composition segregation. Similarly, TEM BF micrographs, SADPs and EDS maps of CoCrN/Mo composites are shown in Fig. 8. The nanoscale phases were found in the microstructure (Fig. 8a) further, SADPs analysis of the phases that were taken along the $[1 \overline{2} 1 \overline{3}]$ zone axes, confirmed that the phases adopted HCP structure. Qualitative chemical analysis of the nanoscale $\mu$ phases was also performed using STEM-EDS. The corresponding STEM-EDS elemental mapping images for different constituent elements are shown in Fig. 8(b1) to (b4). There $\mu$ phases were apparently enriched in $\mathrm{Cr}$ and Mo, but depleted in Co 
and $\mathrm{Ni}$, which is in accordance with SEM-EDS results.

\subsection{Mechanical deformation behavior}

The tensile properties of CoCrNi MEAs with and without Mo addition at room temperature were investigated, and the stress-strain curves are shown in Fig. 9. CoCrNi MEAs exhibited superior fracture strain (53.6\%) but moderate strength (yield strength $(\mathrm{YS})=352 \mathrm{MPa}$, ultimate yield strength $(\mathrm{UTS})=799 \mathrm{MPa})$. Addition of Mo nanoparticles into the MEA enhanced strength and decreased ductility, CoCrNi/Mo composites have high strength $(\mathrm{YS}=815 \mathrm{MPa}$, UTS $=958 \mathrm{MPa})$ but moderate fracture strain $(27.1 \%)$. Moreover, the associated hardness was improved from 159 (0 wt.\% Mo) to $375 \mathrm{HV}$ (5 wt.\% Mo). The fracture surfaces of the tensile test specimens were observed by SEM (Figs. 9b and d). As is shown, dimples with features of typical ductile fracture were observed in CoCrNi MEAs and CoCrNi/Mo composites. In addition, nanoscale particle and many voids left on the surface of the composite after $\mu$ phases were pulled out. In Fig. 9c, the tensile yield strength and fracture strain of the CoCrNi MEAs and $\mathrm{CoCrNi} / \mathrm{Mo}$ composites of the present work are compared with those of the same MEAs synthesized by other methods [7, 11], and with other typical HEAs/MEAs [30-36]. It is seen that CoCrNi MEAs have relative good ductility but moderate yield strength, while $\mathrm{CoCrNi} / \mathrm{Mo}$ composites have superb yield strength but moderate ductility.

TEM micrographs of CoCrNi MEAs and CoCrNi/Mo composites after the tensile deformation are shown in Fig. 10. A high density of dislocations can be seen in CoCrNi MEAs (Fig. 10a). Furthermore, some narrow deformation twins in Fig. 10b with a width of tens of nanometers were observed. Therefore, the plastic deformation of the CoCrNi MEAs was dominantly accommodated by dislocation motion and deformation-induced twinning. In addition, a high density of dislocations and the interaction of dislocations with nanoscale $\mu$ phases were also found in the microstructure of $\mathrm{CoCrNi} / \mathrm{Mo}$ composites. These $\mu$ phases block a large amount of dislocations, as a consequence, forming the pileup of dislocations. Continuing deformation results the bow-out of the pinned segment of dislocations. It is noted that 
$\mu$ phases is not plastically sheared by gliding dislocations during plastic deformation. These non-shearable $\mu$ phases accounts for high strength of CoCrNi/Mo composites according to precipitation strengthening mechanism.

\section{Discussion}

As shown in Table 1, addition of Mo nanoparticles delivered excellent comprehensive mechanical properties. In the following sections, the possible strengthening mechanisms, including: (1) solid solution hardening; (2) load transfer from FCC matrix to $\mu$ phases; (3) Grain refining caused by pinning effect; (4) Orowan strengthening mechanism and (5) Thermal mismatch mechanism resulted from the generation of dislocations due to the different coefficient of thermal expansion (CTE) between FCC matrix and the $\mu$ phases, will be discussed separately:

(1) Solid solution hardening. Among the four elements in Co-Cr-Ni-Mo system, Mo has the largest metallic radius $(\mathrm{Co}(0.125 \mathrm{~nm}), \mathrm{Cr}(0.128 \mathrm{~nm}), \mathrm{Ni}(0.124 \mathrm{~nm})$, Mo $(0.139 \mathrm{~nm}))$. Hence, the current MEA system can be treated as a CoCrNi solvent matrix containing Mo solutes, and thus potentially maximizes solid solution strengthening. A standard model for substitutional solid solution strengthening, based on dislocation-solute elastic interactions, may be applied directly to evaluate the role of solution strengthening caused by Mo using the following relationship [19]:

$$
\Delta \sigma S_{(\mathrm{Mo})}=M \times \frac{G c^{0.5} \varepsilon_{s}^{1.5}}{700}
$$

where $G$ is the shear modulus of CoCrNi matrix. In this study, $G$ can be calculated as $G=E / 2(1+u)$, where $u$ is the Poisson's ration $(\approx 0.33), E$ is the elastic modulus. The value of elastic modulus for CoCrNi MEA, introduced by Ref. [37], is $226 \mathrm{GPa}, c$ of the atomic ratio of Mo is $3.10 \%$ (Calculated by mass ratio) and $M=3.06$ is the Taylor factor (3.06 for both FCC and BCC polycrystalline materials). The interaction parameter $\varepsilon_{s}$ is expressed as:

$$
\varepsilon_{s}=\left|\frac{\varepsilon_{G}}{1+0.5 \varepsilon_{G}}-3 \varepsilon_{\alpha}\right|
$$

It combines the elastic effect and atomic size mismatch, i.e. $\varepsilon_{G}$ and $\varepsilon_{\alpha}$, which are defined respectively as: 


$$
\varepsilon_{G}=\frac{1}{G} \frac{\partial G}{\partial c}, \varepsilon_{\alpha}=\frac{1}{\alpha} \frac{\partial \alpha}{\partial c}
$$

while the effect of $\varepsilon_{G}$ is relatively negligible in comparison with $\varepsilon_{\alpha}$. Thus, the interaction parameter, $\varepsilon_{s}$ is approximately equal to $\left|\frac{3}{\alpha} \frac{\partial \alpha}{\partial c}\right|$, where $\alpha$ is the lattice constant of CoCrNi matrix. The parameter $\varepsilon_{\alpha}$ is readily obtained from the XRD patterns where the lattice parameters are $0.352 \mathrm{~nm}$ and $0.353 \mathrm{~nm}$ for the CoCrNi MEAs and CoCrNi/Mo composites, respectively. Accordingly, the strength enhancement caused by solid solution hardening in the MEA with Mo addition is estimated as $\sim 80.34 \mathrm{MPa}$.

(2) The load transfer effect. In this study, the nanohardness of FCC phase and $\mu$ phase were detected by nanoindentation. The result indicated that the nanohardness of $\mu$ phase was up to $28.72 \mathrm{GPa}$, which is higher than the nanohardness of CoCrNi MEA (8.47 GPa). Therefore, the load can transfer from matrix FCC phases to the uniform distributed $\mu$ phases efficiently during deformation. The improvement in load transfer effect $\Delta \sigma_{L T}$ can be expressed by the equation as following [38]:

$$
\Delta \sigma_{L T}=f_{v} \sigma_{0} / 2
$$

Where $f_{v}$ denotes the volume fraction of $\mu$ phase, $\sigma_{0}$ is the yield strength of CoCrNi MEA. In this work, we adopted the value of $f_{v}$ of $\mu$ phase is $4.6 \%$ (For convenience, the volume fraction of $\mu$ phase was calculated by XRD.), $\sigma_{0}$ of CoCrNi matrix is 352 $\mathrm{MPa}$. Consequently, the yield strength increment from load transfer effect of WC is evaluated as 8.1 MPa.

(3) Strengthening by grain refinement. EBSD result shows that the average grain size of FCC matrix decreased obviously after adding Mo nanoparticles, as shown in Fig. 6. In-situ $\mu$ phases located at grain boundaries can bring a strong drag force between two adjacent grains. Then grain boundaries motion can be inhibited by the pining effect of $\mu$ phases distributing in grain boundaries during sintering, achieving improved mechanical properties. The part of strengthening effects by grain refinement $\left(\Delta \sigma_{G R}\right)$ can be calculated via the Hall-Petch relationship, showing as the following formula [39]: 


$$
\Delta \sigma_{G R}=K\left(d^{-0.5}-d_{0}^{-0.5}\right)
$$

where $K$ is a constant $\left(265 \mathrm{MPa} \cdot \mu \mathrm{m}^{1 / 2}\right.$ for CoCrNi MEA [37]), $d$ and $d_{0}$ are the average grain sizes of $\mathrm{CoCrNi} / \mathrm{Mo}$ composite and raw CoCrNi MEA, respectively. As a consequent, the strength contribution from grain refinement to yield strength is evaluated as 32.01 MPa. Besides, smaller grain size offers a higher volume fraction of grain boundaries, which could further impede dislocation motion. Both effects are the primary reasons for improving mechanical properties of $\mathrm{CoCrNi} / \mathrm{Mo}$ composite by grain refinement.

(4) Orowan strengthening. The fine $\mu$ phases have the size distribution of a dominant number of particles less than $400 \mathrm{~nm}$. During deformation, $\mu$ phases act as hard phase at grain boundaries and in the FCC matrix, which interact with dislocations through the pining effect and block the dislocations movement further (Orowan-type), as shown in Fig. 10d. Slip trace within a single grain can move forward when dislocations bypass $\mu$ phases. Orowan strengthening can impose a great contribution to the improvement in tensile strength for $\mathrm{CoCrNi} / \mathrm{Mo}$ composite, which can be calculated by Orowan-Ashby equation [40]:

$$
\begin{gathered}
\Delta \sigma_{\text {Orowan }}=0.13 G b / \lambda \ln \frac{D}{2 b} \\
\lambda=D\left[\left(2 V_{P}\right)^{-1 / 3}-1\right]
\end{gathered}
$$

where $G$ and $b$ represent the shear modulus and Burgers vector of matrix $\mathrm{CoCrNi}$ MEA, while $D, \lambda$, and $V p$ represent the average diameter, average inter-particulate spacing and volume fraction of $\mu$ phases, respectively. In this study, shear modulus $(G)$ can be calculated as $G=E / 2(1+u)$, where $u$ is the Poisson's ration $(\approx 0.33), E$ is the elastic modulus. The value of elastic modulus for CoCrNi MEA, introduced by Ref. [37], is $226 \mathrm{GPa}$. The magnitude of the Burgers vector is $|b|=a_{0} / \sqrt{2}$ with $a_{0}$ being the lattice constant. The average diameter of $\mu$ phases was measured by Image $\mathbf{J}$ software is $0.17 \mu \mathrm{m}$ (Statistical data based on Fig. 6d), volume fraction of $\mu$ phases is $4.6 \%$ and lattice constant $a_{0}$ detected by XRD is $3.53 \times 10^{-9} \mathrm{~m}$. Consequently, the yield strength increment from Orowan strengthening is evaluated as $77.65 \mathrm{MPa}$. 


\section{(5) Thermal mismatch enhancement}

The thermal mismatch mechanism in composite is bound up with the difference of coefficient of thermal expansion between the matrix and the reinforcement phase [41, 42]. In this study, the coefficient of thermal expansion (CTE) of $\mu$ phase and $\mathrm{CoCrNi}$ MEA are $8 \times 10^{-6} \mathrm{~K}^{-1}$ (refer to $\gamma$ phase) [43] amd $17.4 \times 10^{-6} \mathrm{~K}^{-1}$ [44], respectively. Thus, the new thermal mismatch dislocations may be generated at the matrix $-\mu$ phase interfaces during deformation to relax thermal stresses. The contributions of CTE mismatch strengthening to the yield strength can be expressed as follows [45].

$$
\begin{gathered}
\Delta \sigma_{C T E}=\eta G b \rho^{1 / 2} \\
\rho=12 \Delta \alpha \Delta T V_{P} /\left[b D\left(1-V_{P}\right)\right]
\end{gathered}
$$

where $\eta$ is a constant equal to 1.25 [46], and $\rho$ is the enhanced dislocation density. $\Delta \alpha$ is the difference in the CTEs of the matrix $\left(17 \times 10^{-6} \mathrm{~K}^{-1}\right)$ and $\mu$ phase $\left(8 \times 10^{-6}\right.$ $\mathrm{K}^{-1}$ ). $\Delta T$ is the difference between the processing temperature (solidification temperature of $\left.1200{ }^{\circ} \mathrm{C}\right)$ and the test temperature $\left(25^{\circ} \mathrm{C}\right)$. Thus, the contribution of thermal mismatch (WC nanoparticles) is evaluated as $173.4 \mathrm{MPa}$.

In summary, the calculated values of the quantitative contribution from solid solution hardening, load transfer effect, grain refinement, Orowan strengthening and thermal mismatch enhancement are 80.34, 8.10, 32.01, 77.65 and $173.4 \mathrm{MPa}$, respectively. Solid solution hardening and thermal mismatch enhancement are much more effective than other strengthening mechanisms. In addition, the calculated yield strength, 723.5 MPa, is lower than the measured yield strength of 815.0 MPa. The discrepancy may be attributable to some parameters used for calculation are approximations, or cited from other HEAs.

\section{Conclusions}

In this study, CoCrNi/Mo composites were fabricated by a combination of novel coating method and SPS. The following conclusions can be dawn:

(a) Compared with ex-situ Mo particles, CoCrNi/Mo mixed powder synthesized by coating method exhibited superior advantages, including distribution uniformly, 
on oxidation and contamination.

(b) The significant increase in strength was achieved in CoCrNi/Mo composites, the yield strength increased from 352 to $815 \mathrm{MPa}$ and the hardness improved from 159 to $375 \mathrm{HV}$ for CoCrNi MEAs without and with 5\% Mo nanoparticles.

(c) The improved properties are mainly attributed to synergistic effects of various strengthening mechanisms. The calculated values of the quantitative contribution from solid solution hardening, load transfer effect, grain refinement, Orowan strengthening and thermal mismatch enhancement were 80.34, 8.10, 32.01, 77.65 and 173.4 MPa, respectively.

\section{Acknowledgments}

This project was financially supported by National Natural Science Foundation of China with No. 51404302.

\section{References}

[1] J.W. Yeh, Recent progress in high-entropy alloys, Eur. J. Contr. 31 (2006) 633-648.

[2] A. Gail, E.P. George, Tensile properties of high- and medium-entropy alloys, Intermetallics 39 (2013) 74-78.

[3] Z. Fu, W. Chen, Z. Chen, H. Wen, E.J. Lavernia, Influence of Ti addition and sintering method on microstructure and mechanical behavior of a medium-entropy $\mathrm{Al}_{0.6} \mathrm{CoNiFe}$ alloy, Mater. Sci. Eng., A 619 (2014) 137-145.

[4] E.W. Huang, D.J. Yu, J.W. Yeh, C. Lee, K. An, A study of lattice elasticity from low entropy metals to medium and high entropy alloys, Scripta Mater. 101 (2015) $32-35$.

[5] V.T. Nguyen, M. Qian, Z. Shi, T. Song, L. Huang, A novel quaternary equiatomic Ti-Zr-Nb-Ta medium entropy alloy (MEA), Intermetallics 101 (2018) 39-43.

[6] Z. Li, K.G. Pradeep, Y. Deng, D. Raabe, C.C. Tasan, Metastable high-entropy dual-phase alloys overcome the strength-ductility trade-off, Nature 534 (2016) $227-230$.

[7] B. Gludovatz, A. Hohenwarter, K.V.S. Thurston, H. Bei, Z. Wu, Exceptional 
damage-tolerance of a medium-entropy alloy $\mathrm{CrCoNi}$ at cryogenic temperatures, Nat. commun. 7 (2016) 10602-10609.

[8] Y.L. Zhao, T. Yang, Y. Tong, J. Wang, J.H. Luan, Heterogeneous precipitation behavior and stacking-fault-mediated deformation in a CoCrNi-based medium-entropy alloy, Acta Mater. 138 (2017) 72-82.

[9] Y. Ma, F. Yuan, M. Yang, P. Jiang, E. Ma, Dynamic shear deformation of a CrCoNi medium-entropy alloy with heterogeneous grain structures, Acta Mater. 148 (2018) $407-418$.

[10] S. Yoshida, T. Bhattacharjee, B. Yu, N. Tsuji, Friction stress and Hall-Petch relationship in CoCrNi equi-atomic medium entropy alloy processed by severe plastic deformation and subsequent annealing, Scripta Mater. 134 (2017) 33-36.

[11] I. Moravcik, J. Cizek, Z. Kovacova, J. Nejezchlebova, M. Kitzmantel, Mechanical and microstructural characterization of powder metallurgy CoCrNi medium entropy alloy, Mater. Sci. Eng., A 701 (2017) 370-380.

[12] Y. Lu, Y. Dong, S. Guo, L. Jiang, H. Kang, A Promising New Class of High-Temperature Alloys: Eutectic High-Entropy Alloys, Sci. Rep. 4 (2014) 6200.

[13] Z. Li, D. Raabe, Influence of compositional inhomogeneity on mechanical behavior of an interstitial dual-phase high-entropy alloy, Mater. Chen. Phys. 210 (2017) 29-36.

[14] T. Niendorf, T. Wegener, Z. Li, D. Raabe, Unexpected cyclic stress-strain response of dual-phase high-entropy alloys induced by partial reversibility of deformation, Scripta Mater. 143 (2018) 63-67.

[15] Y.F. Kao, T.J. Chen, S.K. Chen, J.W. Yeh, Microstructure and mechanical property of as-cast,-homogenized, and-deformed $\mathrm{Al}_{\mathrm{x}} \mathrm{CoCrFeNi}(0 \leqslant \mathrm{x} \leqslant 2)$ high-entropy alloys, J. Alloys Comp. 488 (2009) 57-64.

[16] W.H. Liu, J.Y. He, H.L. Huang, H.Wang, Z.P. Lu, Effects of Nb additions on the microstructure and mechanical property of $\mathrm{CoCrFeNi}$ high-entropy alloys, Intermetallics 60 (2015) 1-8.

[17] W.H. Liu, Z.P. Lu, J.Y. He, J.H. Luan, Z.J. Wang, Ductile $\mathrm{CoCrFeNiMo}_{\mathrm{x}}$ high entropy alloys strengthened by hard intermetallic phases, Acta Mater. 116 (2016) 
$332-342$.

[18] T.T. Shun, L.Y. Chang, M.H. Shiu, Age-hardening of the $\mathrm{CoCrFeNiMo}_{0.85}$ high-entropy alloy, Mater. Charact. 81 (2013) 92-96.

[19] J.Y. He, H. Wang, H.L. Huang, X.D. Xu, M.W. Chen, A precipitation-hardened high-entropy alloy with outstanding tensile properties, Acta Mater. 102 (2016) $187-196$.

[20] L. Zhang, Y. Zhou, X. Jin, X. Du, B. Li, Precipitation-hardened high entropy alloys with excellent tensile properties, Mater. Sci. Eng., A 732 (2018) 186-191

[21] J.Y. Wang, H.L. Yang, Z.L. Liu, S.X. Ji, R.D. Li, J.M. Ruan, A novel $\mathrm{Fe}_{40} \mathrm{Mn}_{40} \mathrm{Cr}_{10} \mathrm{Co}_{10} / \mathrm{SiC}$ medium-entropy nanocomposite reinforced by the nanoparticles-woven architectural structures, J. Alloys Comp. 772 (2019) 272-279.

[22] Q.C. Fan, B.S. Li, Y. Zhang, The microstructure and properties of $(\mathrm{FeCrNiCo}) \mathrm{Al}_{\mathrm{x}} \mathrm{Cu}_{\mathrm{y}}$ high-entropy alloys and their TiC-reinforced composites, Mater. Sci. Eng., A 598 (2014) 244-250.

[23] I. Moravcik, L. Gouvea, J. Cupera, I. Dlouhy, Preparation and properties of medium entropy CoCrNi/boride metal matrix composite, J. Alloys Comp. 748 (2018) 979-988.

[24] A. Raza, H.J. Ryu, S.H. Hong, Strength enhancement and density reduction by the addition of $\mathrm{Al}$ in $\mathrm{CrFeMoV}$ based high-entropy alloy fabricated through powder metallurgy, Mater. Des. 157 (2018) 97-104.

[25] M. He, J.Y. Wang, R.G. He, H.L. Yang, J.M. Ruan, Effect of cobalt content on the microstructure and mechanical properties of coarse grained WC-Co cemented carbides fabricated from chemically coated composite powder, J. Alloys Comp. 766 (2018) 556-563.

[26] R.G. He, J.Y. Wang, M. He. H.L. Yang, J.M. Ruan, Synthesis of WC composite powder with nano-cobalt coatings and its application in WC-4Co cemented carbide, Ceram. Int. 44 (2018) 10961-10967.

[27] X.Q. Chen, H.X. Zhang, W.H. Shen, Preparation and characterization of the magnetic Fe3O4@TiO2 nanocomposite with the in-situ synthesis coating method, Mater. Chem. Phys. 216 (2018) 496-501. 
[28] C.F. Sun, P.P. Li, S.Q. Xi, Y. Zhou, S.W. Li, A new type of high entropy alloy composite $\mathrm{Fe}_{18} \mathrm{Ni}_{23} \mathrm{Co}_{25} \mathrm{Cr}_{21} \mathrm{Mo}_{8} \mathrm{WNb}_{3} \mathrm{C}_{2}$ prepared by mechanical alloying and hot pressing sintering, Mater. Sci. Eng., A 728 (2018) 144-150.

[29] J. Chen, P.Y. Niu, T. Wei, L. Hao, Y.Z. Liu, Fabrication and mechanical properties of $\mathrm{AlCoNiCrFe}$ high-entropy alloy particle reinforced $\mathrm{Cu}$ matrix composites, J. Alloys Comp. 15 (2015) 630-634.

[30] B. Gludovatz, A. Hohenwarter, D. Catoor, E.H. Chang, E.P. George, A fracture-resistant high-entropy alloy for cryogenic applications, Science 345 (2014) $1153-1158$.

[31] W. Huo, H. Zhou, F. Fang, X. Hu, Z. Xie, Strain-rate effect upon the tensile behavior of CoCrFeNi high-entropy alloys, Mater. Sci. Eng., A 689 (2017) 366-369.

[32] Y. Deng, C.C. Tasan, K.G. Pradeep, H. Springer, A. Kostka, Design of a twinning-induced plasticity high entropy alloy, Acta Mater. 94 (2015) 124-133.

[33] T. Bhattacharjee, R. Zheng, Y. Chong, S. Sheikh, S. Guo, Effect of low temperature on tensile properties of $\mathrm{AlCoCrFeNi} \mathrm{Ci}_{2.1}$ eutectic high entropy alloy, Mater. Chem. Phys. 210 (2018) 207-212.

[34] C.J. Tong, Y.L. Chen, J.W. Yeh, S.J. Lin, S.K. Chen, Microstructure characterization of $\mathrm{Al}_{x} \mathrm{CoCrCuFeNi}$ high-entropy alloy system with multiprincipal elements, Metall. Mater. Trans. A 36 (2005) 881-893.

[35] K. Ming, X. Bi, J. Wang, Precipitation strengthening of ductile $\mathrm{Cr}_{15} \mathrm{Fe}_{20} \mathrm{Co}_{35} \mathrm{Ni}_{20} \mathrm{Mo}_{10}$ alloys, Scripta Mater. 137 (2017) 88-93.

[36] Z. Li, K.G. Pradeep, Y. Deng, D. Raabe, C.C. Tasan, Metastable high-entropy dual-phase alloys overcome the strength-ductility trade-off, Nature 534 (2016) $227-230$.

[37] S. Yoshida, T. Bhattacharjee, B. Yu, N. Tsuji, Friction stress and Hall-Petch relationship in CoCrNi equi-atomic medium entropy alloy processed by severe plastic deformation and subsequent annealing, Scripta Mater. 134 (2017) 33-36.

[38] A. Corporation, Carbon Nanotubes Reinforced Metal Matrix Composites.

[39] N. Hansen, Hall-Petch relation and boundary strengthening, Scripta Mater. 51 (2004) 801-806. 
[40] F. Chen, Z.N. Chen, F. Mao, T.M. Wang, Z.Q. Cao, TiB 2 reinforced aluminum based in situ composites fabricated by stir casting, Mater. Sci. Eng., A 625 (2015) $357-368$.

[41] M.M. Toropova, C.A. Steeves, Adaptive bimaterial lattices to mitigate thermal expansion mismatch stresses in satellite structures, Acta Astronaut. 113 (2015) $132-141$.

[42] W.D. Fei, M. Hu, C.K. Yao, Thermal expansion and thermal mismatch stress relaxation behaviors of $\mathrm{SiC}$ whisker reinforced aluminum composite, Mater. Chem. Phys. 77 (2003) 882-888.

[43] Y.N. Gornostyrev, O.Y. Kontsevoi, K.Y. Khromov, M.I. Katsnelson, A.J. Freeman, The role of thermal expansion and composition changes in the temperature dependence of the lattice misfit in two-phase $\gamma / \gamma^{\prime}$ superalloys, Scripta Mater. 56 (2007) $81-84$.

[44] I. Moravcik, J. Cizek, Z. Kovacova, J. Nejezchlebova, M. Kitzmantel, Mechanical and microstructural characterization of powder metallurgy CoCrNi medium entropy alloy, Mater. Sci. Eng., A 701 (2017) 370-380.

[45] F. Chen, Z.N. Chen, F. Mao, T.M. Wang, Z.Q. Cao, TiB 2 reinforced aluminum based in situ composites fabricated by stir casting, Mater. Sci. Eng., A 625 (2015) $357-368$.

[46] Z.W. Liu, N. Cheng, Q.L. Zheng, J.H. Wu, Q.Y. Han, Z.F. Huang, J.D. Xing, Y.F. Li, Y.M. Gao, Processing and tensile properties of A356 composites containing in situ small-sized $\mathrm{Al}_{3}$ Ti particulates, Mater. Sci. Eng., A 710 (2018) 392-399. 


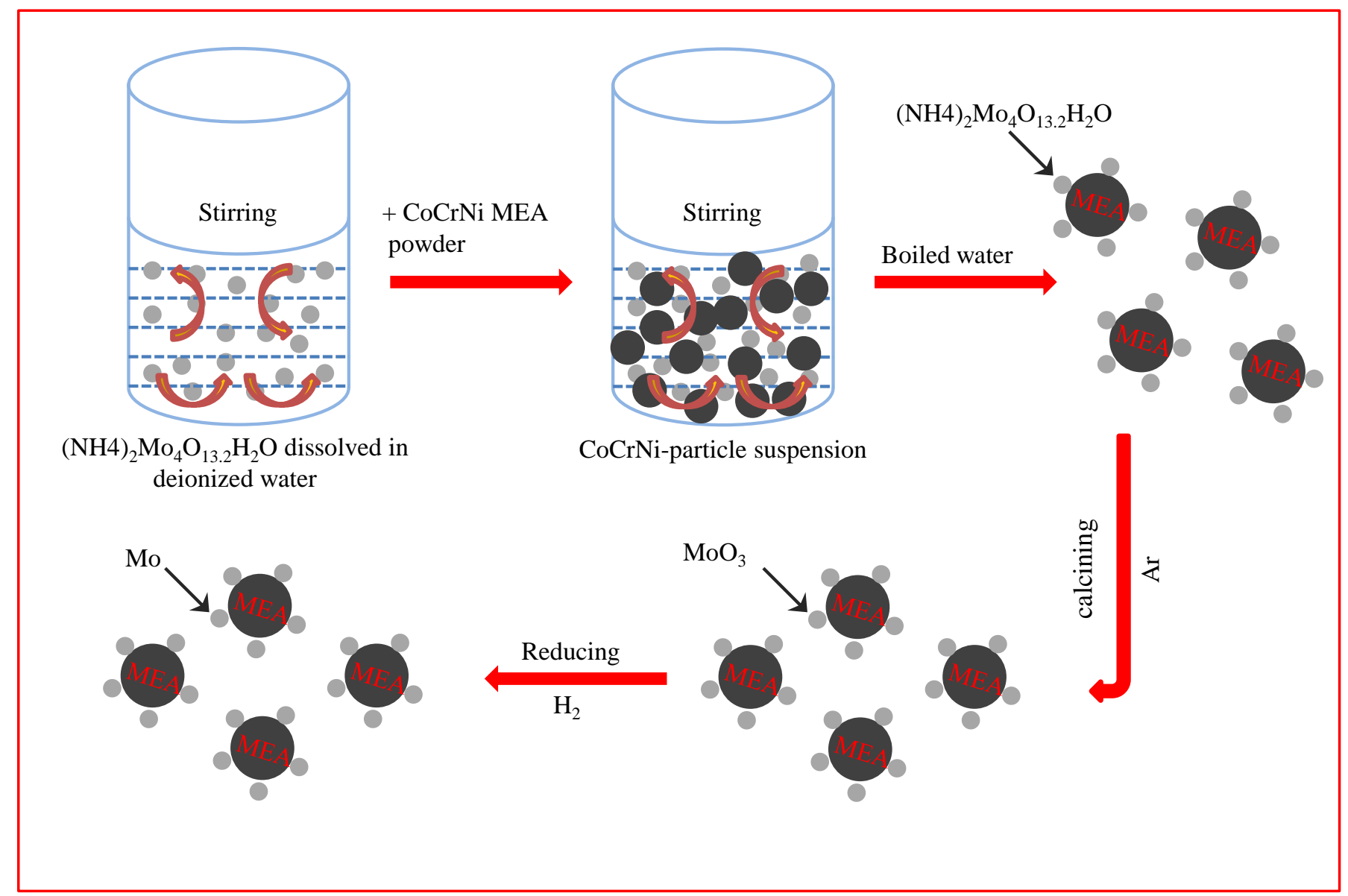

Fig. 1. Schematic diagram of synthesis procedure for $\mathrm{CoCrNi} / \mathrm{Mo}$ mixed powder by coating method. 

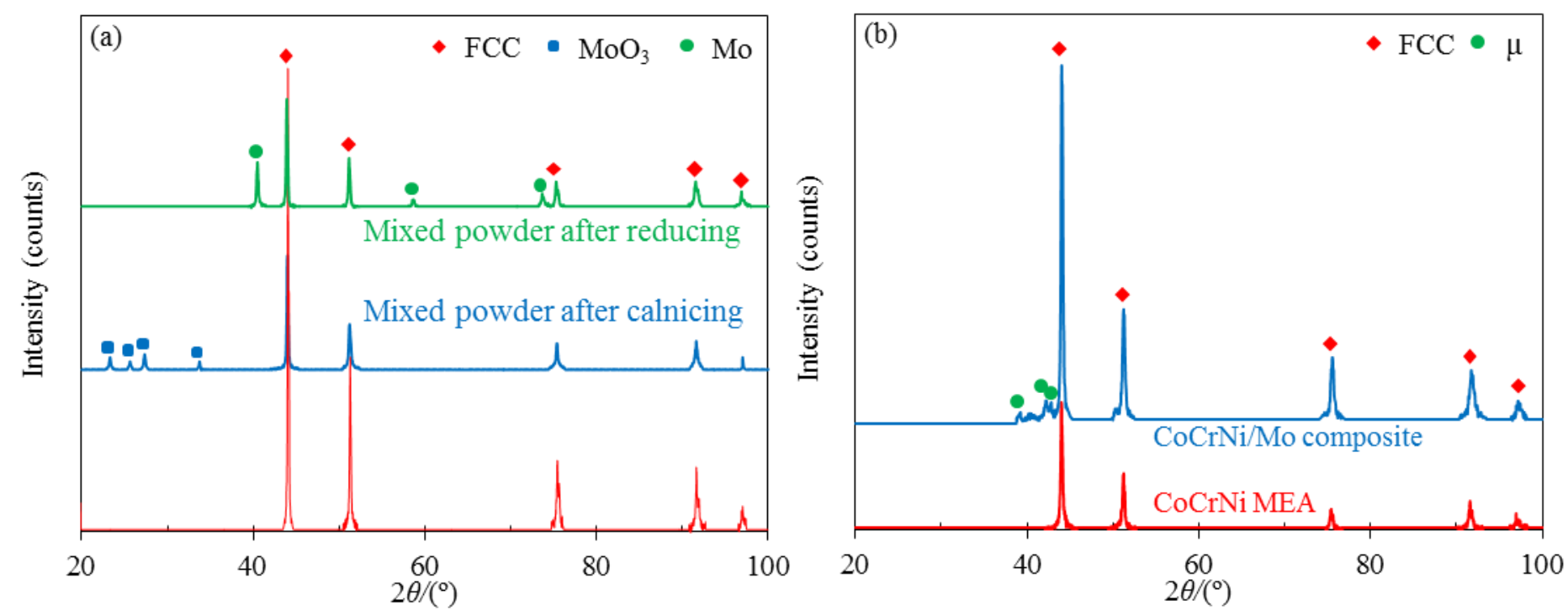

Fig. 2. XRD spectra of (a) mixed powder at different states and (b) CoCrNi MEAs and CoCrNi/Mo composites after SPS. 

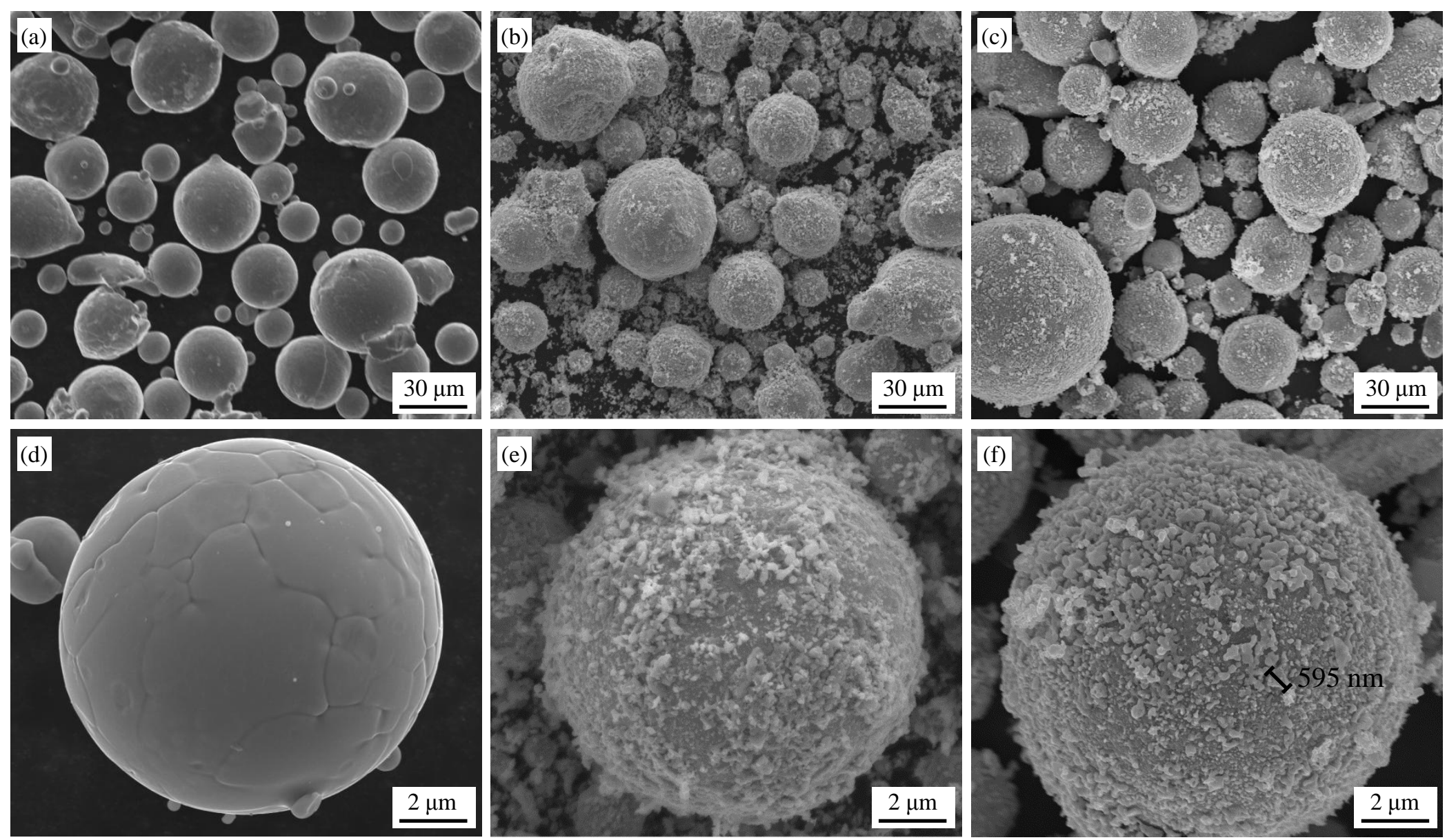

Fig. 3. SEM micrographs showing the surfaces of mixed powder at different states. (a) and (d) the pure CoCrNi gas atomized powder; (b) and (e) mixed powder after calcining; (c) and (f) mixed powder after reducing. Black marking in (f) indicates that the maximum size of reduced Mo particle does not exceed $\sim 600 \mathrm{~nm}$. 

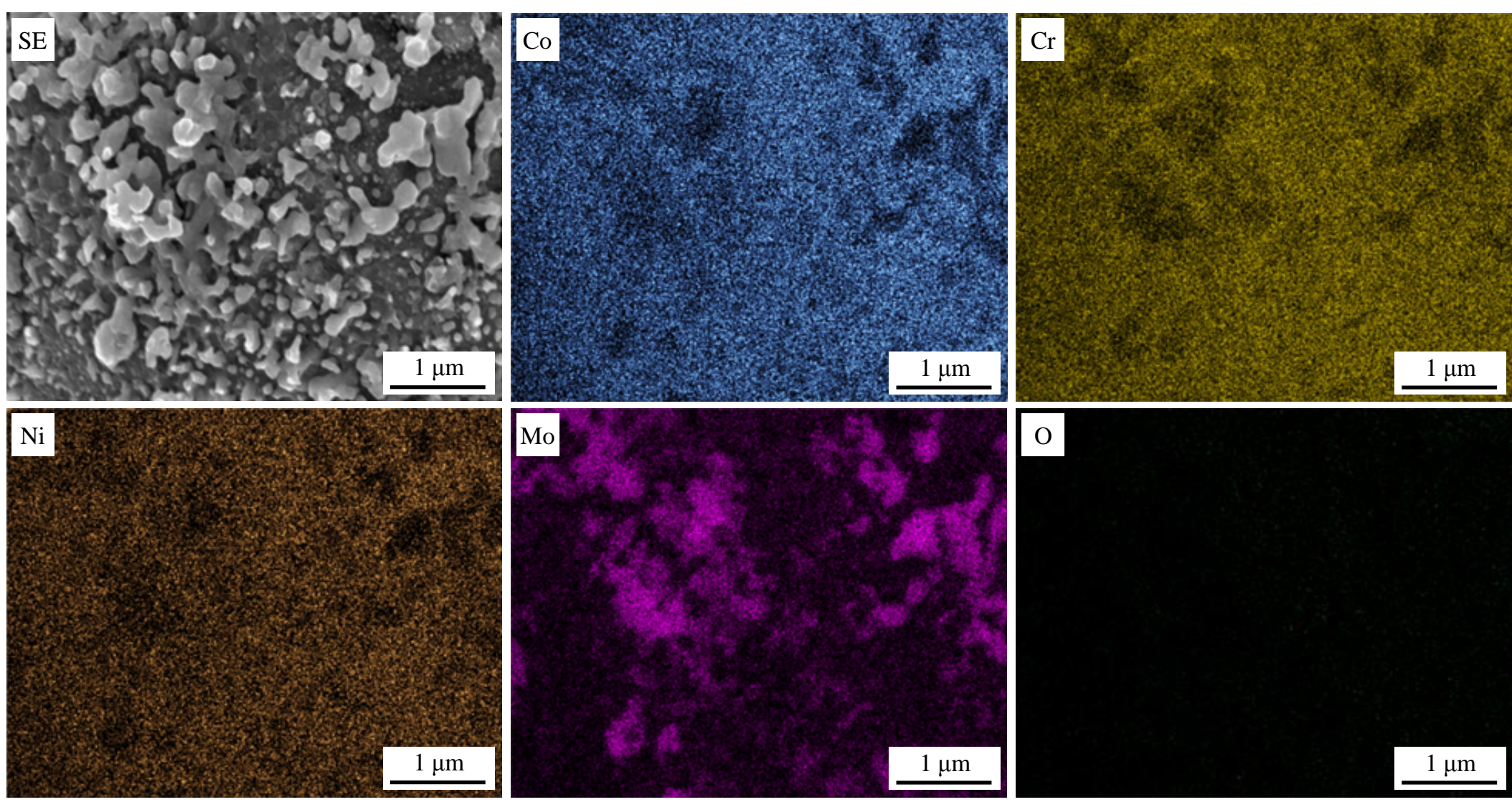

Fig. 4. SEM/EDS mapping showing the element distribution of the $\mathrm{CoCrNi} / \mathrm{Mo}$ mixed powder after reducing. 

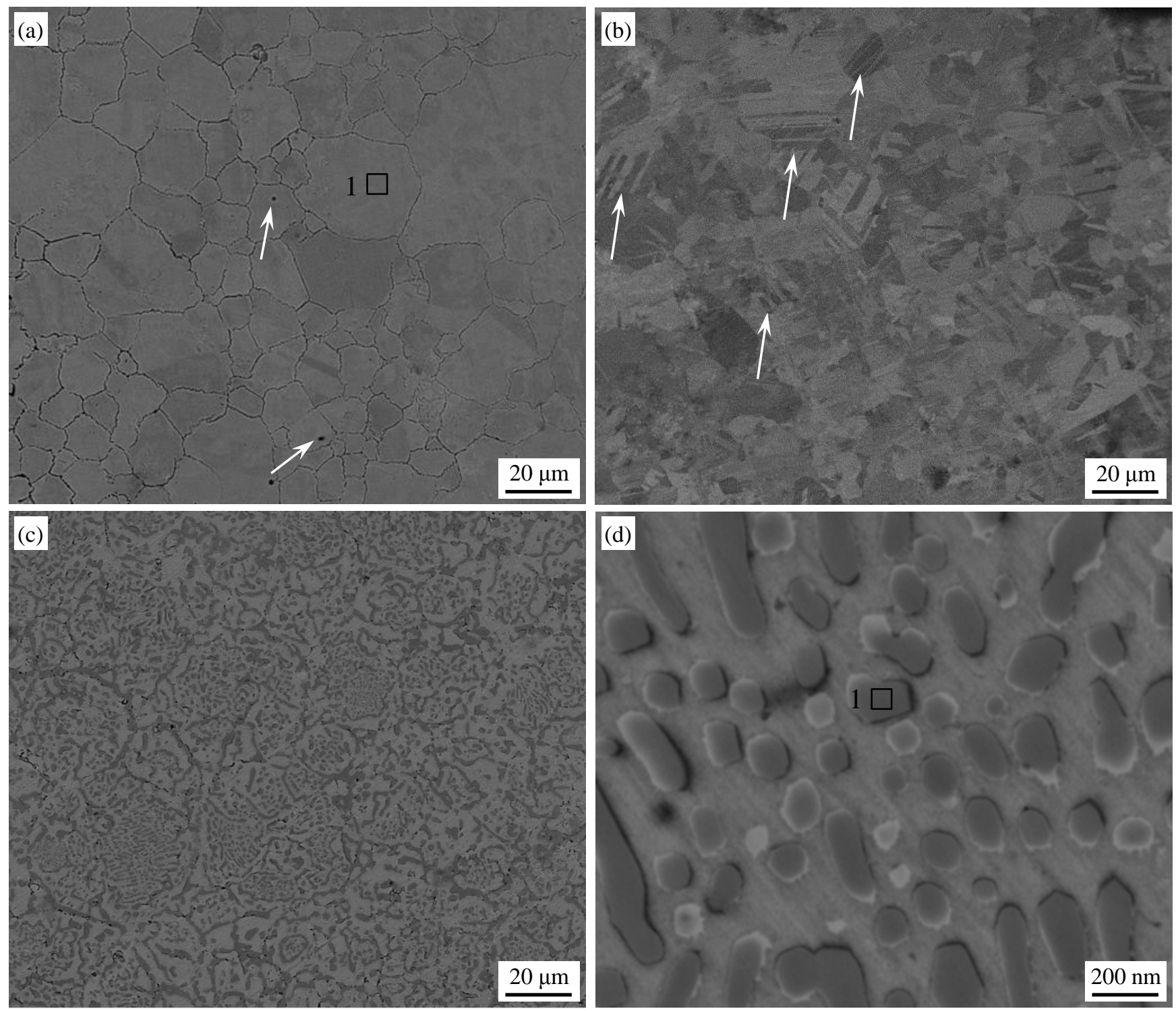

Fig. 5. Backscattered SEM micrographs showing the microstructure of CoCrNi MEAs (a and b) and CoCrNi/Mo composites (c and d). White arrows in (a) and (b) indicate some visible porosities and twinning phases, respectively. 


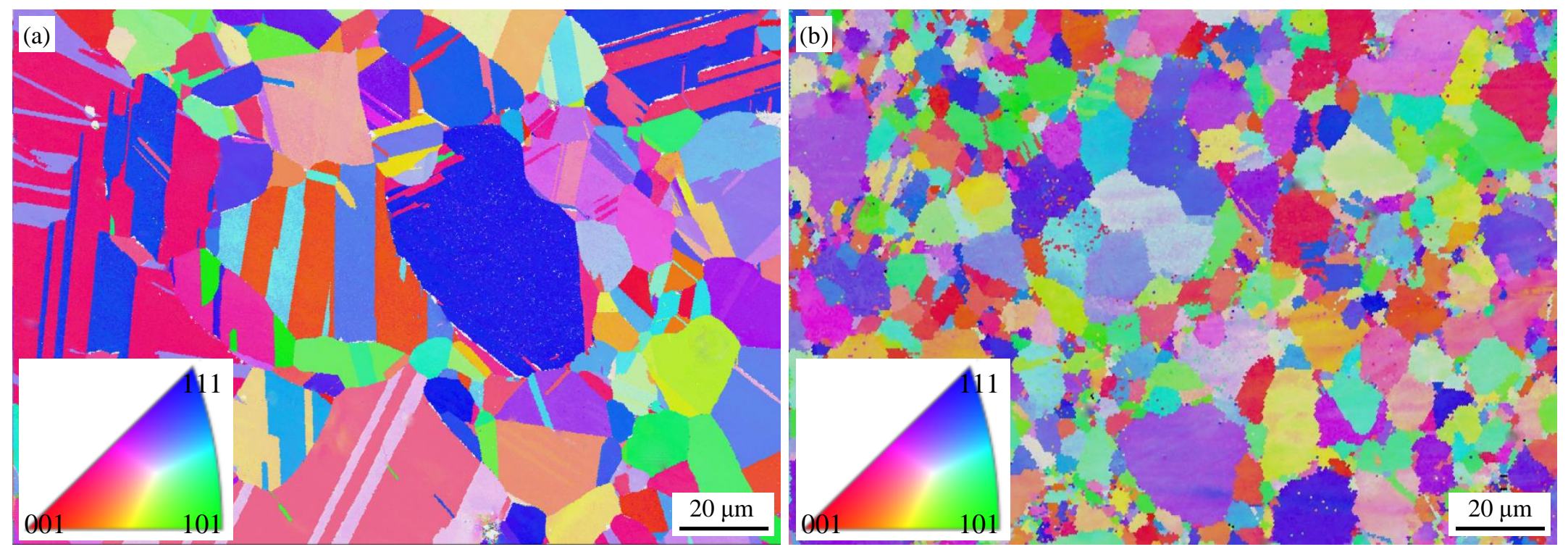

Fig. 6. EBSD mapping-based microstructure showing the variation of grain sizes of (a) CoCrNi MEAs and (b) $\mathrm{CoCrNi} / \mathrm{Mo}$ composites. The inserts in (a) and (b) are the inverse pole figures (IPFs) of the FCC phase in the alloy. 


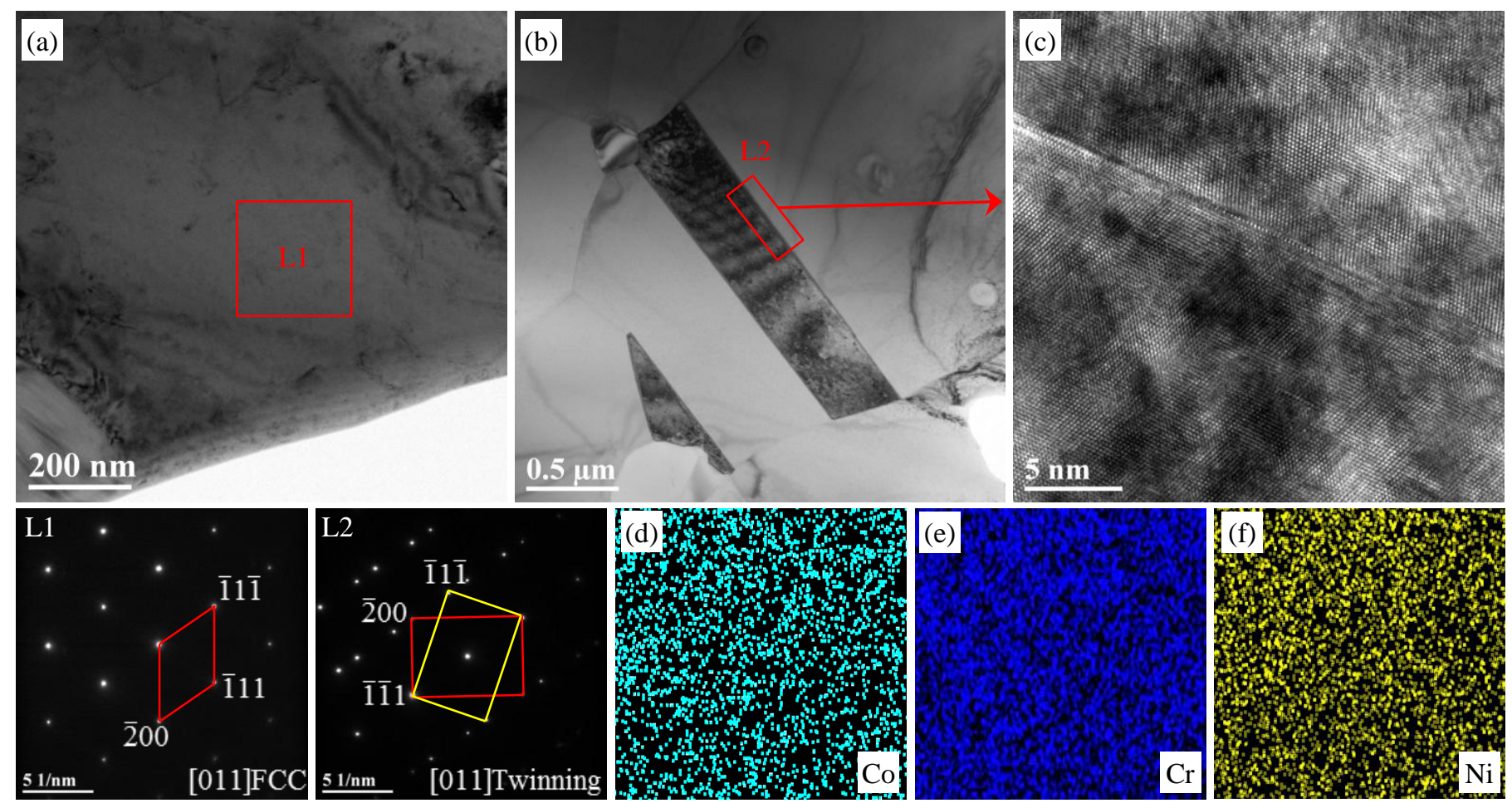

Fig. 7. TEM analysis of CoCrNi MEAs, including BF (a) and SADP (L1) showing FCC structure of CoCrNi MEAs; BF (b), HRTEM (c) and SADP (L2) showing annealing twins. (d)-(f) show the corresponding EDS maps of area L1 in (a). 


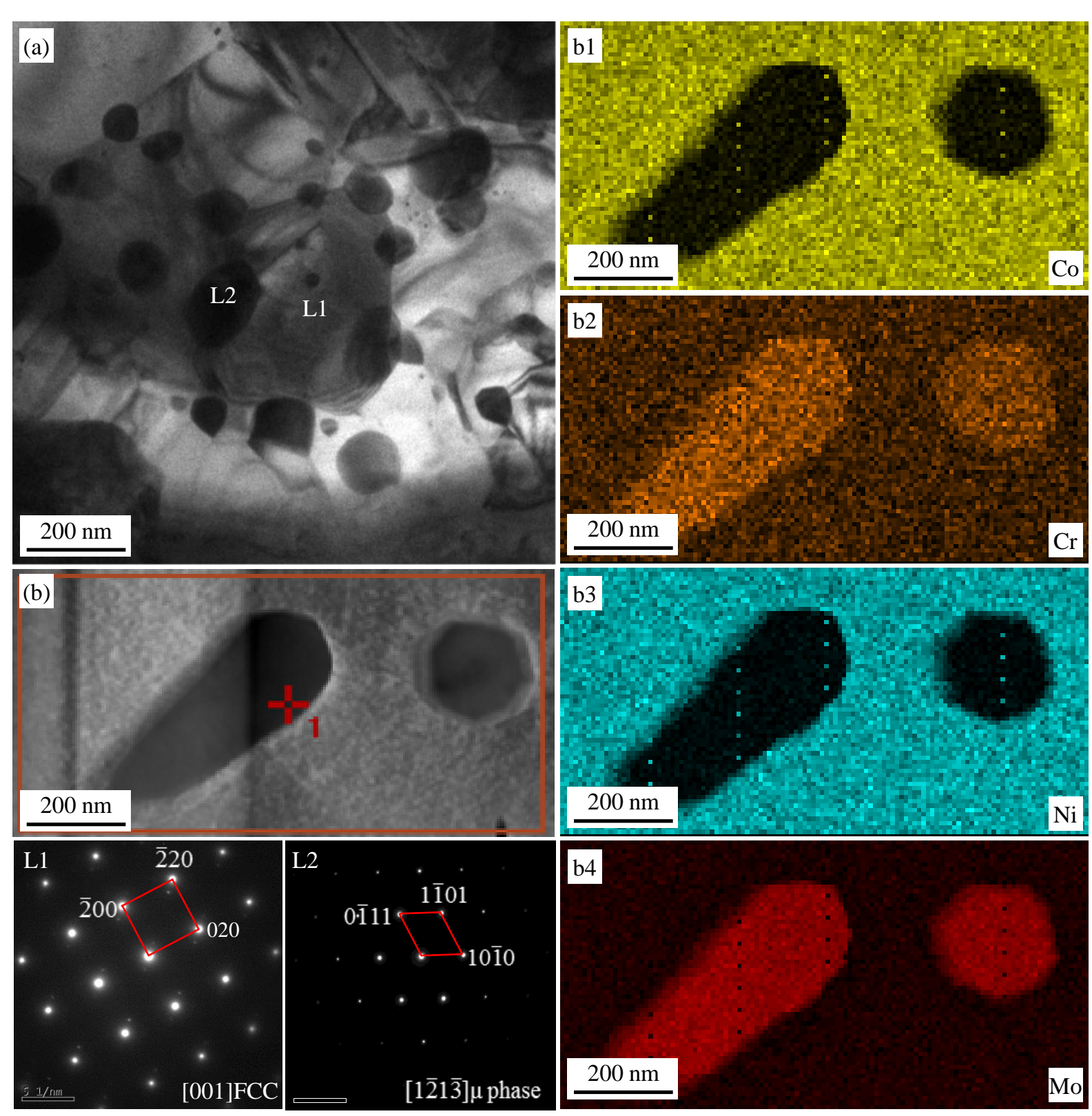

Fig. 8. TEM analysis of CoCrNi/Mo composites, including BF (a) and SADP (L1 and L2) showing FCC structure and $\mu$ phase, respectively; (b1)-(b4) show the corresponding EDS maps of (b), including elements of (b1) Co, (b2) Cr, (b3) Ni and (b4) Mo. 

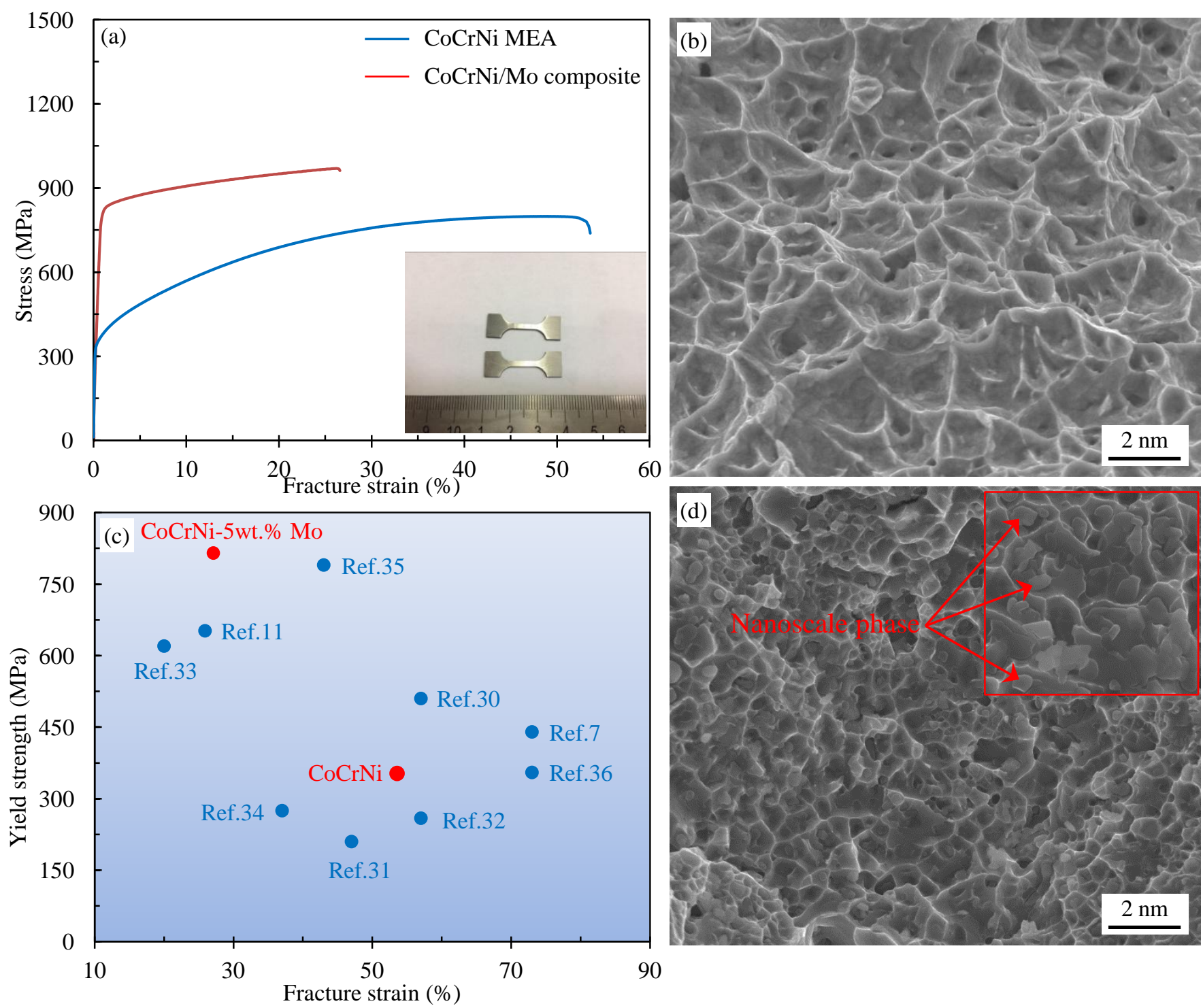

Fig. 9. (a) Tensile stress-strain curves of the pure CoCrNi MEAs and CoCrNi/Mo composites; (b and d) Fracture surfaces of as-sintered CoCrNi MEAs and and $\mathrm{CoCrNi} / \mathrm{Mo}$ composites, respectively; (c) Comparison of strength vs deformation of CoCrNi MEAs synthesized by other methods [7, 11], other typical HEA/MEAs [30-36]. 

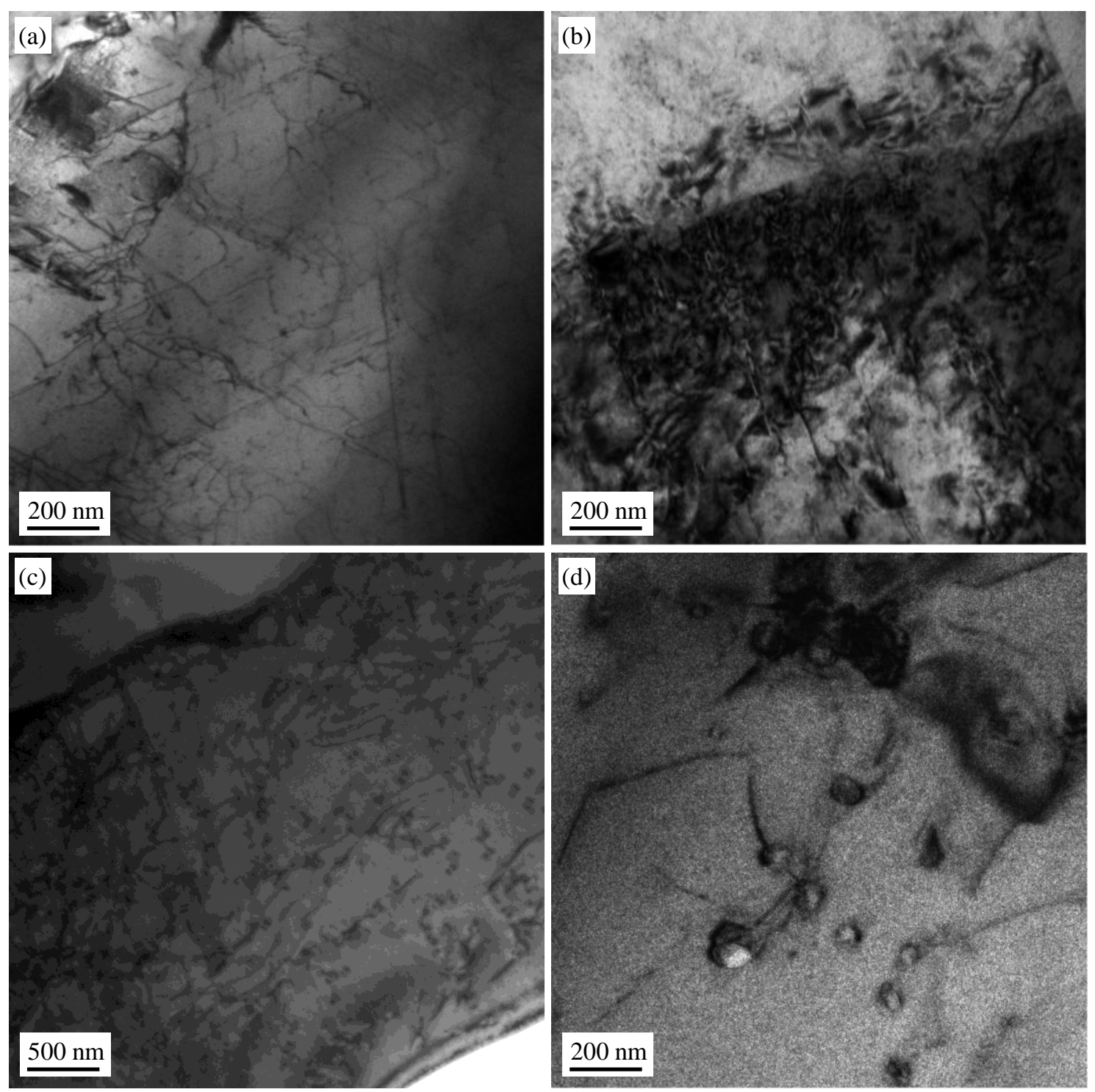

Fig. 10. TEM micrographs of the sintered CoCrNi MEAs (a and b) and CoCrNi/Mo composites (c and d) after the tensile deformation, (a) a high density of dislocations and (b) deformation nanotwins in CoCrNi MEAs; (c) a high density of dislocation and (d) the interaction of dislocation with nanoscale $\mu$ phases in $\mathrm{CoCrNi} / \mathrm{Mo}$ composites. 
Table 1. Measured chemical composition (at.\%) from EDS analysis in different regions.

\begin{tabular}{|c|c|c|c|c|c|}
\hline Characterization method & Atomic $\%$ & & & & \\
\hline & Co & $\mathrm{Cr}$ & $\mathrm{Ni}$ & Mo & $\mathrm{O}$ \\
\hline EDS (mapping Fig. 4) & 32.19 & 30.26 & 31.58 & 5.28 & 0.69 \\
\hline EDS (point Fig. 5a) & 32.72 & 34.08 & 32.84 & - & 0.36 \\
\hline EDS (point Fig. 5d) & 11.08 & 35.79 & 15.03 & 37.85 & 0.25 \\
\hline EDS (mapping Fig. 7) & 32.43 & 34.25 & 32.89 & - & 0.43 \\
\hline EDS (mapping Fig. 8) & 12.34 & 36.54 & 11.56 & 38.73 & 0.83 \\
\hline
\end{tabular}


Table 2. Mechanical properties YS, UTS, fracture strain and hardness of CoCrNi MEAs and CoCrNi/Mo composites.

\begin{tabular}{ccccc}
\hline & Yield strength (YS, MPa) & $\begin{array}{c}\text { Ultimate yield strength } \\
(\text { UTS, MPa })\end{array}$ & fracture strain $(\%)$ & Hardness (HV) \\
\hline CoCrNi & 352 & 799 & 53.6 & 159 \\
$\mathrm{CoCrNi} / \mathrm{Mo}$ & 815 & 958 & 27.1 & 375 \\
\hline
\end{tabular}




\section{Graphical abstract}
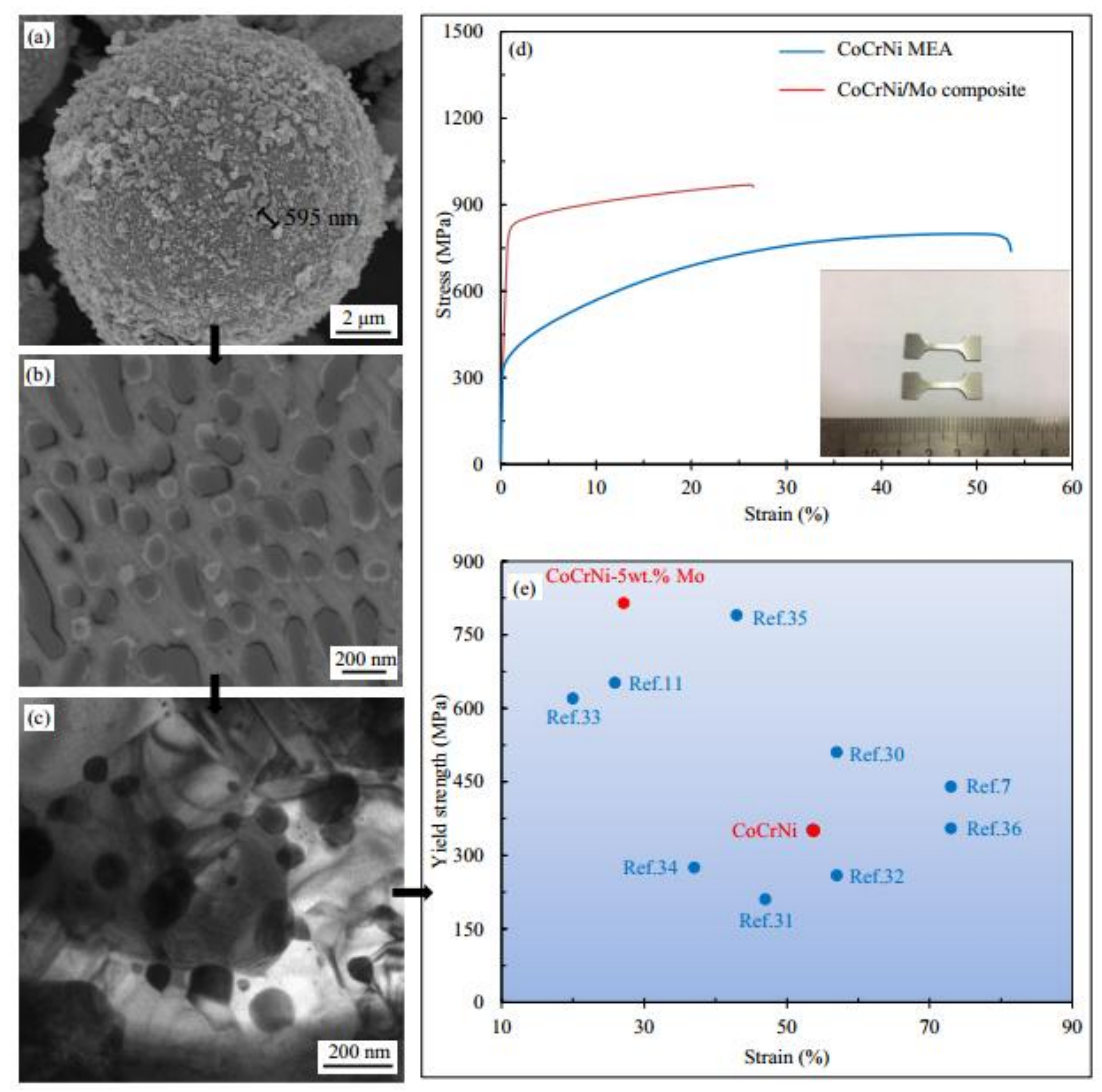

The CoCrNi/Mo composites were fabricated by a combination of novel coating method and spark plasma sintering (SPS) for the first time. The improved properties of composite are mainly attributed to synergistic effects of various strengthening mechanisms caused by in-situ $\mu$ phases. The yield strength increases from 352 to 815 $\mathrm{MPa}$ and the hardness improves from $159 \mathrm{HV}$ to $375 \mathrm{HV}$ for CoCrNi MEA without and with 5\% Mo particles. 


\section{Highlights}

- The CoCrNi/Mo composites were produced by a combination of novel coating method and spark plasma sintering for the first time.

- The as-SPSed composites possess excellent yield strength of $815 \mathrm{MPa}$ and moderate elongation of $27.1 \%$.

- The improved properties are mainly attributed to synergistic effects of various strengthening mechanisms, especially thermal mismatch mechanism.

- This method could give insights on the application of other MEA or HEA-based composites fabricated by chemically coated composite powder. 